\title{
ANÁLISIS MORFOMÉTRICO DE DOLINAS Y PARÁMETROS GEOFÍSICOS APLICADOS AL ESTUDIO DE LOS FLUJOS DE AGUA SUBTERRÁNEA EN LA CUENCA DEL EBRO, ZARAGOZA, ESPAÑA
}

\author{
MORPHOMETRIC ANAL YSIS OF DOLINES AND GEOPHYSICAL PARAMETERS \\ APPLIED TO THE STUDY OF UNDERGROUND WATER FLOWS IN THE EBRO BASIN, \\ ZARAGOZA, SPAIN
}

\author{
Fernando MORAIS ${ }^{1} \&$ Maria Asunción SORIANO² \\ Universidade Federal do Tocantins. Rua 03, Q.17, s/n - Setor Jardim dos Ipês, Porto Nacional - TO, CEP 77500-000 \\ Endereço eletrônico: morais@uft.edu.br \\ (2) Departamento de Ciencias de la Tierra, Facultad de Ciencias, Universidad de Zaragoza, Calle Pedro Cerbuna, n. 12, 50009, \\ Zaragoza, España. Endereço eletrônico: asuncion@unizar.es
}

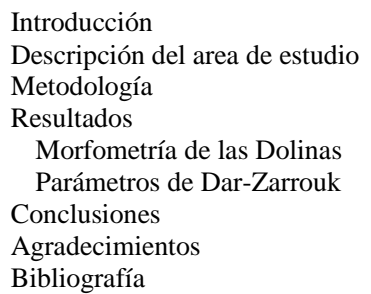

RESUMEN - El relieve kárstico constituye una excelente herramienta para la comprensión de la dinámica del paisaje, además de proporcionar huellas sobre la paleogeografía y paleoecología del planeta. La región de Zaragoza, nordeste de España, presenta una gran cantidad de dolinas aluviales siendo una de las zonas más afectadas por procesos kársticos en la Península Ibérica. En la cuenca central del Ebro el karst en evaporitas a menudo causa daños en carreteras, edifícios y líneas de agua. El objetivo deste trabajo fue investigar posibles relaciones entre la distribución de las dolinas y los flujos hídricos subterráneos. Así que se llevó a cabo el análisis morfométrico de dolinas además de la determinación de los parámetros geofísicos de Dar-Zarrouk, buscando establecer posibles vínculos entre las formas kársticas, flujos hídricos y aspectos geológicos y geomorfológicos de la zona de estudio. Los resultados indicaron predomínio de formas pequeñas, con distribución controlada por estruturas geológicas tales cómo fallas y fracturas. Las zonas con valores intermedios y altos de conductancia longitudinal además de valores intermedios de resistencia transversal presentan mayor concentración de dolinas. Esta relación puede apuntar áreas más propícias al desarrollo de colapsos kársticos en la región estudiada.

Palabras clave: Karst, Dolinas, Sondeos Eléctricos Verticales, Parámetros de Dar-Zarrouk, Cuenca del Ebro.

\begin{abstract}
RESUMO - O relevo cárstico constitui uma excelente ferramenta para a compreensão da dinâmica da paisagem, além de fornecer indícios da paleogeografia e paleoecologia do planeta. A região de Zaragoza, nordeste da Espanha, apresenta uma grande quantidade de dolinas aluviais, sendo uma das áreas mais afetadas por processos cársticos na Península Ibérica. Na bacia do rio Ebro o carste em evaporitos tem causado danos às estradas, edifícios e rede de distribuição de água. O objetivo deste foi investigar possíveis relações entre a distribuição das dolinas e os fluxos de água subterrânea. Para tanto, foi realizada a análise morfométrica das dolinas, juntamente com a determinação dos parâmetros geofísicos de Dar-Zarrouk, buscando estabelecer possíveis vínculos entre a feições cársticas, os fluxos hídricos e aspectos geológicos e geomorfológicos da área de estudo. Os resultados indicaram predominância de feições pequenas, tendo sua distribuição controlada por estruturas geológicas, tais como falhas e fraturas. As áreas com valores de condutância longitudinal intermediários a altos, juntamente com valores intermediários de resistência transversal, apresentaram maior concentração de dolinas. Esta relação pode apontar áreas mais propícias à formação de colapsos cársticos na região estudada.
\end{abstract}

Palavras-chave: Carste, Dolinas, Sondagem Elétrica Vertical, Parâmetros de Dar-Zarrouk, Bacia do Ebro.

\section{INTRODUCCIÓN}

El estudio del relieve kárstico permite una amplia comprensión tanto de la fisiografía como de la dinámica de los paisajes actuales además de aportar huellas sobre la paleogeografía y paleoclimatología del planeta. Los procesos hidrológicos subterráneos son de gran importancia para la génesis y dinámica de los sistemas kársticos (De Waele et al., 2009), independientemente de su composición litológica. En el modelado kárstico el proceso de disolución de las rocas es responsable por generar una capacidad integrada de permitir circulación de fluidos en el sistema. Así que, el agua juega un papel importante en la génesis y evolución de este tipo de relieve.

Las dolinas son consideradas formas diagnósticas de karst (Sweeting, 1972; Ford \& Williams, 1989; Summerfield, 1991; Sauro, 2003) e, incluso, como las formas fundamentales en este contexto geomorfológico. Desde el trabajo pionero de Cvijić (1893) las dolinas son clasificadas en función de su forma $y$ procesos genéticos, aúnque determinar el origen de estas formas puede 
ser muy difícil dependiendo de la cantidad de factores, tales como los geológicos y geomorfológicos, que contribuyen a su génesis (Beck, 1986; Jennings, 1987). Estas depresiones kársticas son mejor comprendidas cuando las estudiamos a través de análisis de sus procesos hidrológicos (Day, 1976; Sinclair et al., 1985), siendo el análisis morfométrico un excelente método para la comprensión de estos procesos (Williams, 1971, 1972, 1983; Ogden, 1988; Denizman, 2003; Brinkmann et al., 2008).

Además del análisis morfométrico, otros métodos han sido utilizados para entender los factores que intervienen en la génesis $\mathrm{y}$ evolución de las dolinas. Entre éstos, los métodos geofísicos han sido aplicados para determinar la geometría interna y los flujos hídricos en estas formas kársticas (Arandjelovic, 1966, 1969; Stevanovic \& Dragisic, 1998; Ahmed \& Carpenter, 2003; Stierman, 2004; Milanovic, 2004; Kruse et al., 2006; Veress, 2009; Valois et al., 2011; Land, 2013; Carbonel et al., 2014).

Para la caracterización de los flujos hídricos subterráneos hay una gran variedad de métodos, como los trazadores, los modelos matemáticos de líneas equipotenciales y los ensayos clásicos de bombeo en pozos. La geofísica desde hace tiempo ha sido aplicada para a ubicación de pozos, para definición de franja de contaminación, además para la determinación del interfaz agua salada/agua dulce, y otras aplicaciones. Los métodos geofísicos se pueden organizar en geoeléctrico, sísmica, gravimetria, magnetometría y geotérmicos (Reynolds, 2011). Entre estos, los métodos geoeléctricos son los más utilizados en los estudios hidrogeológicos, y la adopción de los sondeos eléctricos verticales (VES), y perfiles eléctricos son las técnicas más utilizadas.
Por sus características geológicas, la parte central de la cuenca del río Ebro es una de las zonas más afectadas por procesos kársticos en la Península Ibérica (Gil et al., 2012). Esta región ha sido estudiada desde perspectivas puramente geológicas hasta abordage de planificación ambiental y análisis de riesgos (Benito, 1987; Benito \& Gutiérrez, 1988; Soriano, 1990; Soriano \& Simón, 1995; Gutierrez-Santolalla et al., 2005; Gutiérrez et al., 2008; Luzón et al., 2008, 2012; Galve et al., 2009; Pueyo-Anchuela et al., 2010).

Dado que los índices morfométricos de las dolinas pueden ser utilizados como indicadores de los procesos kársticos responsables de su génesis (Palmquist, 1979; Bondesan et al., 1992), y los parámetros geofísicos de DarZarrouk pueden ser utilizados como indicadores de los flujos del agua subterránea (Singh et al., 2004; Srinivas et al., 2012), los principales objetivos de este estudio son: (i) determinar la distribución espacial de las dolinas en un sector cercano a la ciudad de Zaragoza, España; (ii) calcular los parámetros morfométricos de dichas dolinas; (iii) calcular los parámetros geofísicos de Dar-Zarrouk para el área de estudio y (iv) analizar cualitativamente la relación entre la distribución y la morfometría de las dolinas y el comportamiento del flujo de agua subterránea, predichos a partir de los parámetros geofísicos calculados. Esas informaciones son muy importantes para la comprension de la génesis de dolinas en sedimentos evaporíticos, que según Gutiérrez et al. (2004) constituye aproximadamente $7 \%$ del território de España, y es menos conocido que las áreas de calizas. Además, en áreas donde no hay disponibilidad de informaciones hidrogeológicas $\mathrm{o}$ en proyectos con presupuestos limitados, dichos parámetros son de grande valia a la comprensión de los flujos subterráneos.

\section{DESCRIPCIÓN DEL AREA DE ESTUDIO}

El área estudiada se encuentra en la parte central de la Cuenca Terciaria del Ebro (NE de España), que es la cuenca de antepaís meridional de la cordillera de los Pirineos (Riba et al., 1983). La cuenca también está limitada por la Cordillera Ibérica hacia el sur y la Cordillera Costera Catalana hacia el este. En la actualidad, el río Ebro atraviesa esta cuenca y fluye hacia el este hacia el Mar Mediterráneo. En su parte central, el espesor de la serie Paleógeno y Neógeno llega a 1500 m (Riba et al., 1983). Hasta el Eoceno Tardío, la cuenca fue conectada con el Océano Atlántico. Más tarde, se convirtió en sistemas endorreicos y abanicos aluviales con evaporitas poco profundas y sistemas lacustres en carbonatos 
desarrollados (Muñoz et al., 2002; Pardo et al., 2004). La mayor parte de las rocas expuestas corresponden a evaporitas terrígenas (yeso y halita principalmente) y facies carbonatadas depositados en estos ambientes. La Cuenca del Ebro se abrió hacia el mar Mediterráneo por el Mioceno tardío. El sistema de drenaje desarrollado, posteriormente erosionado, favoreció la sedimentación fluvial y aluvial en el Cuaternario.

Los cambios en el nivel de base hicieron que el desarrollo de terrazas con niveles escalonados. Recientes estudios sedimentológicos y geomorfológicos en algunas de las terrazas fluviales del Pleistoceno en el Ebro (Luzón et al., 2008, 2012; Gil et al., 2013) han demons- trado que fueron depositadas principalmente en un sistema fluvial entrelazado. Además, espesores anómalos y facies raras en tal entorno fluvial son comunes e interpretado estar relacionado con sinsedimentarias actividad kárstica (Benito et al., 1998; Luzón et al., 2008; Gil et al., 2013).

Presencia de conjuntos de fracturas es frecuente tanto, en las rocas del Neógeno y en los depósitos cuaternarios suprayacentes (Arlegui \& Soriano, 1996). Aunque éstos muestran una gran dispersión, orientaciones NS y WNW-ESE dominan el sistema. Estas fracturas han condicionado el desarrollo del sistema de drenaje y la mayoría de las formas de relieve en esta cuenca (Arlegui \& Soriano, 1996).

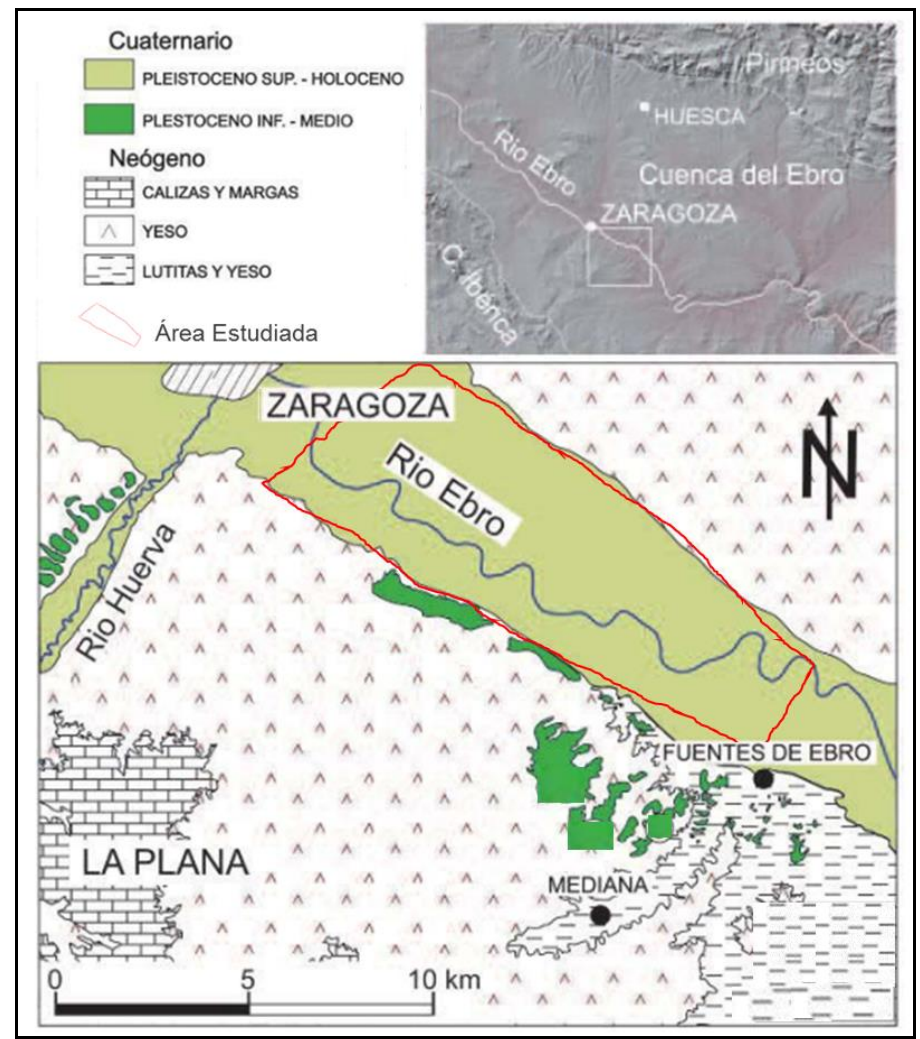

Figura 1- Geología y localización del área estudiada (Modificado de Gil et al., 2013)

En la actualidad, las inundaciones, los movimientos de pendiente y karstificación son algunos de los principales procesos geológicos en la cuenca central del Ebro. Sin embargo, la karstificación en evaporitas, probablemente, es el proceso que causa más importante advertencia sociales allí. Alrededor de 700.000 habitantes viven en esta área y, con frecuencia, las zonas urbanas se ven afectados por el desarrollo de dolinas con daños posteriores en carreteras, edificios, líneas de agua, etc.
(Soriano \& Simón, 1995; Pueyo-Anchuela et al., 2010).

Existen numerosos registros de desarollo de dolinas en sítios dentro de la cuenca aluvial del Ebro, alcanzando densidades de hasta 22 formas por kilómetro cuadrado, que se considera muy alta (Soriano et al., 1994), teniendo en cuenta la literatura sobre el tema. En el aluvial del río Ebro, estas formas han sido más destacadas en el tramo entre las localidades de Pinseque y Pina de Ebro, aguas 
arriba y aguas abajo de la ciudad de Zaragoza, respectivamente (Benito, 1987; Soriano, 1992; Gutiérrez et al., 2008) .

Aunque el área de ocurrencia de estas formas kársticas es más amplio (Lamelas, 2007), para este estudio se seleccionó una zona de dolinas con superficie total de $73,76 \mathrm{~km}^{2}$, delimitada por un polígono irregular de aproximadamente $15 \mathrm{~km}$ de largo por $5 \mathrm{~km}$ de ancho, aguas abajo de la ciudad de Zaragoza, España (Figura 1). Esta área fue seleccionada por: (i) presentar gran número de dolinas y (ii) por la disponibilidad de datos de los estudios geofísicos.

\section{METODOLOGÍA}

Los parámetros de Dar-Zarrouk tienen gran importancia para comprender los flujos subterráneos en un medio estratificado (Singh, 2004), ya que resultan de las características espesor y resistividad - de cada capa geoeléctrica. Se definieron por primera vez por Maillet (1947) al estudiar la anisotropía de resistividad de los medios estratificados y que se refieren a la resistencia transversal (T) y la conductancia longitudinal unitaria (S). Para determinar los parámetros de Dar-Zarrouk para una secuencia de $n$ capas horizontales, homogéneas y isotrópicas, la resistividad $\rho i$ y el espesor $h i$, se calcula la resistencia transversal (T) multiplicando la resistividad aparente de cada capa por su respectivo espesor (Ecuación $1)$ :

$T=\rho 1 . h 1+\rho 2 . h 2+\rho 3 . h 3 \ldots \ldots+\rho n . h n=\sum_{i=1}^{N} \rho i . h i$

(Ecuación 1)

La conductancia longitudinal (S) se define a partir de la relación entre los espesores de las capas y sus respectivas resistividades (Ecuación 2):

$$
\mathrm{S}=\frac{\mathrm{h} 1}{\mathrm{p} 1}+\frac{\mathrm{h} 2}{\mathrm{p} 2}+\frac{\mathrm{h} 3}{\mathrm{p} 3} \ldots . .+\frac{\mathrm{hn}}{\mathrm{pn}}=\sum_{\mathrm{i}=1}^{\mathrm{N}} \frac{\mathrm{hi}}{\mathrm{pi}}
$$

(Ecuación 2)

Basándose en la interpretación de los parámetros de Dar-Zarrouk, es posible inferir la ubicación de las zonas con mayor transmisividad de un acuífero, además las direcciones de los flujos subterráneos, lo que permite la generación de mapas potenciométricos. Kelly (1976) señaló que los sondeos eléctricos verticales constituye una base para estimar la conductancia específica de las aguas subterráneas. Este autor encontró una relación empírica entre la resistividad eléctrica del acuífero y su conductividad hidráulica (Kelly, 1977). Basándose en una revisión bibliográfica, es posible observar la relación suficientemente detectable y fiable entre los parámetros Dar-Zarrouk y las características hidráulicas de los acuíferos (Kosinski \& Kelly, 1981; Niwas \& Singhal, 1981, 1985; Niwas et al., 2011).

Cómo ya se ha expuesto, la utilización de parámetros geofísicos y morfométricos para comprender el flujo subterráneo en zonas de dolinas es el objetivo principal de este estudio. Para lograr este objetivo se ha adoptado la siguiente metodologia: (1) Exhaustiva revisión bibliográfica de estudios previos llevados a cabo en la cuenca del Ebro, principalmente en la porción seleccionada entre las desembocaduras de los ríos Gállego y Ginel, aguas abajo de la ciudad de Zaragoza. (2) A partir de la delimitación de las dolinas, hecha por Lamelas (2007), para cada forma se midieron los atributos morfométricos apuntados en la tabla 1. La definición de los parámetros medidos se hizo sobre la base de las obras clásicas de análisis morfométrico de dolinas (Williams, 1972; Palmquist, 1979; Mills \& Starnes, 1983; Bondesan et al., 1992; Denizman, 2003). Se utilizaron los softwares Global Mapper 12.0 y Image J.

En este trabajo se utilizaron los datos de campo de 37 sondeos eléctricos verticales realizados por la Dirección General de Obras Hidráulicas del Ministerio de Obras Públicas y Desarrollo Urbano en España. Los datos fueron cedidos por la Confederación Hidrográfica del Ebro (CHE). Los ensayos se realizaron con un DC eletrorresistivímetro, adoptando la disposición Schlumberger para la distribución de los electrodos, con una abertura máxima entre 40 y $620 \mathrm{~m}$ para los electrodos de corriente. Este tipo de prospección geofísica se resume en introducir en el suelo una corriente eléctrica (I) a través de dos electrodos A y B, conectados a una fuente de energia y medir la tensión $(\Delta \mathrm{V})$ generada en los otros dos eléctrodos, nombrados $\mathrm{M}$ y N, o eléctrodos de potencial (Reynolds, 2011). 
Tabla 1 - Atributos morfométricos analisados en este estudio; basado en Mills \& Starnes (1983) y Denizman (2003).

\begin{tabular}{|c|c|c|}
\hline $\begin{array}{l}\text { Nombre del } \\
\text { Parámetro }\end{array}$ & Definición & Unidad \\
\hline Longitud & Longitud maxima de la forma. & $\mathrm{m}$ \\
\hline Anchura & Anchura maxima de la forma. & $\mathrm{m}$ \\
\hline Area & Area dentro del contorno de la forma. & $\mathrm{m}^{2}$ \\
\hline Perímetro & $\begin{array}{l}\text { Línea que involucra el maximo contorno externo de la } \\
\text { forma. }\end{array}$ & $\mathrm{m}$ \\
\hline Orientación & Orientación geográfica del eje más largo de la dolina. & grados \\
\hline $\begin{array}{l}\text { Indice de } \\
\text { Circularidad }\end{array}$ & $\begin{array}{l}\text { Relación entre el area de la dolina y el area de un } \\
\text { círculo con perímetro idéntico al de la dicha forma. }\end{array}$ & ---- \\
\hline $\begin{array}{l}\text { Relación } \\
\text { Longitud/Anchura }\end{array}$ & $\begin{array}{l}\text { Parámetro derivado de la relación entre longitud y } \\
\text { anchura de las formas }\end{array}$ & ---- \\
\hline Pitting Index & $\begin{array}{l}\text { Relación entre el area total estudiada y la suma de las } \\
\text { areas de las dolinas. }\end{array}$ & ---- \\
\hline
\end{tabular}

Los valores de campo fueron ajustados a los modelos teóricos y a la realidad geológica local. Para ello, se utilizó el software de ajuste automático ABEM Super-VES con el error de menos de 0,5\% en los cálculos (Octavio de Toledo, 1989). Aunque en las interpretaciones posteriores se han utilizado datos locales de estratigrafia, inicialmente las curvas de resistividad se clasificaron según su descomposición en intervalos de tres capas, y cada intervalo permite cuatro posibilidades de curvas (Orellana, 1972):

Tipo $H$ - la segunda capa es la menos resistiva, es decir $\rho 1>\rho 2<\rho 3$;

Tipo $K$ - la segunda capa es la más resistiva, es decir $\rho 1<\rho 2>\rho 3$;

Tipo A - la resistividad aumenta con la profundidad; es decir $\rho 1<\rho 2<\rho 3$;

Tipo $Q$ - la resistividad disminuye con la profundidad; o sea $\rho 1>\rho 2>\rho 3$.

\section{RESULTADOS}

\section{Morfometria de las dolinas}

El área de estudio tiene 318 dolinas dispuestas en un área total de $73,76 \mathrm{~km}^{2}$, lo que resulta en una densidad de 4,31 dolinas $/ \mathrm{km}^{2}$. A partir del análisis de 4 áreas aguas arriba de Zaragoza, Gil et al. (2013) presentan una densidad media de 9,83 dolinas $/ \mathrm{km}^{2}$. Sin embargo, la comparación de los valores de densidad encontrados en diferentes estudios es dificultada por la escala adoptada en la cartografía. Cerca de la desembocadura del río Gallego, Benito et al. (1995) indicaron la existencia de 22 dolinas en un área de $0,25 \mathrm{~km}^{2}$. A partir de observaciones y cartografia de campo a escala 1:1000, Maldonado et al. (2000) encontraron una densidad de 633.39 dolinas $/ \mathrm{km}^{2}$ para esta misma zona.

La longitud de las dolinas se obtiene por medio la medición del eje mayor entre los extremos de la forma kárstica. La distribución simplificada de las longitudes para este estudio muestra una asimetría positiva (Figura 2a). Los valores medidos oscilaron entre 18,3-1.041m, con media de $145,87 \mathrm{~m}$ y desviación estándar de $149,85 \mathrm{~m}$.

Las anchuras de las dolinas están determinadas por la mayor distancia perpendicular a la línea de longitud. Igual que las longitudes, las anchuras presentaron una asimetría positiva en su distribución (Figura 2b). Los valores encontrados tuvieron una variación desde 12,07$450,47 \mathrm{~m}$, con media de $73,07 \mathrm{~m}$ y desviación estándar de 78,46m. La coherencia entre la anchura y la longitud puede ser vista a traves del coeficiente de correlación entre estos dos atributos, que era $\mathrm{R}^{2}=0,75$ (Figura 3 ). 

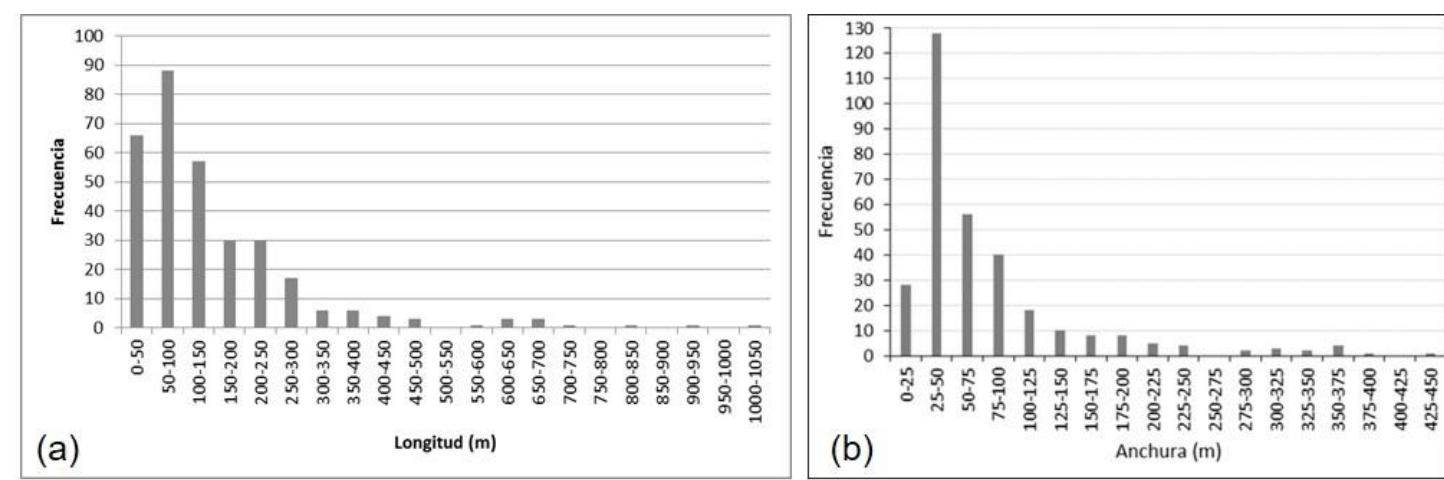

Figura 2- Distribución natural de los valores de longitude (a) y anchura (b) de las dolinas.

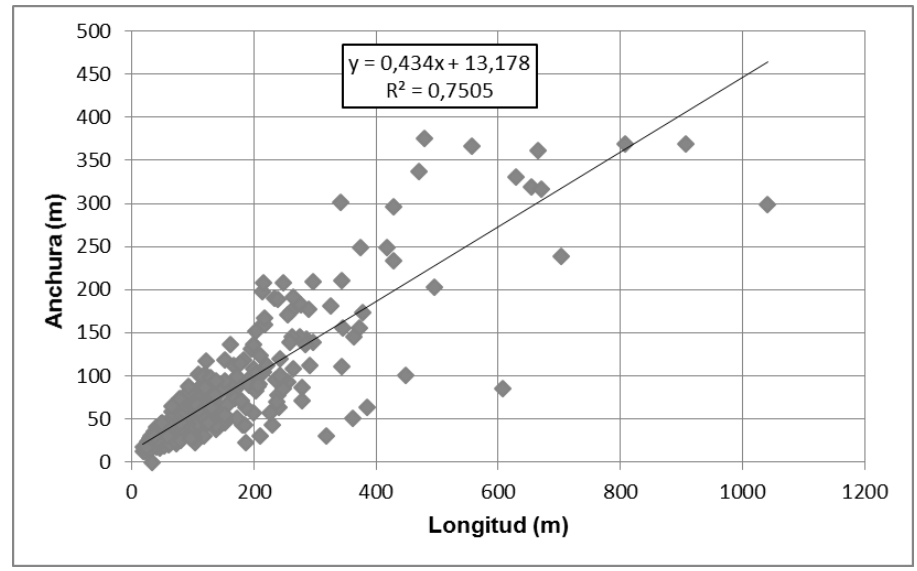

Figura 3- Relación entre longitud y anchura de las dolinas investigadas.

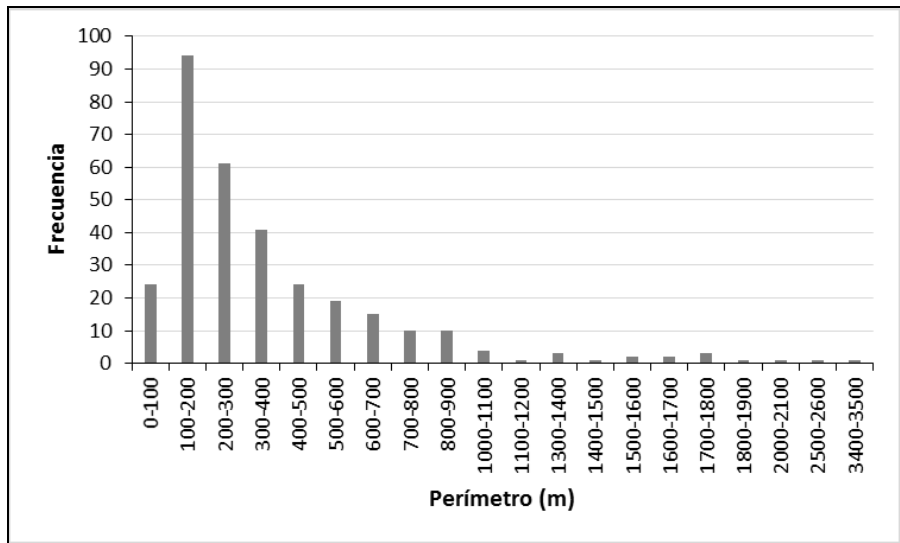

Figura 4- Distribución natural de los perímetros de las dolinas estudiadas

El perímetro de una dolina se define por la extensión de la línea continua al largo del borde exterior de esta forma geomorfológica. Los valores de perímetro econtrados para el área de estudio oscilaron entre 47,76 y $3.435,15 \mathrm{~m}$, con media de 398,86m y desviación estándar de 390,07m. Los perímetros aquí analizados muestran una distribución natural asimétrica positiva (Figura 4). Es necesario indicar que la relación entre perímetro y área de las dolinas no constituye una condición sine qua non para el análisis morfométrico, ya que dicha relación es muy dependiente del grado de irregularidad de los contornos de las formas (Mills \& Starnes, 1983).

Los valores individuales de area de las dolinas en la zona estudiada oscilaron entre 162,68 y $211.713,83 \mathrm{~m}^{2}$, con media de $13.821,95 \mathrm{~m}^{2}$ y desviación estándar de $28.850,97 \mathrm{~m}^{2}$. Al igual que los atributos anteriores, las áreas medidas presentan una distribución natural asimétrica positiva (Figura 5). Estas cifras muestran un predominio de pequeñas dolinas, mientras son pocas las formas con grandes áreas individuales. 


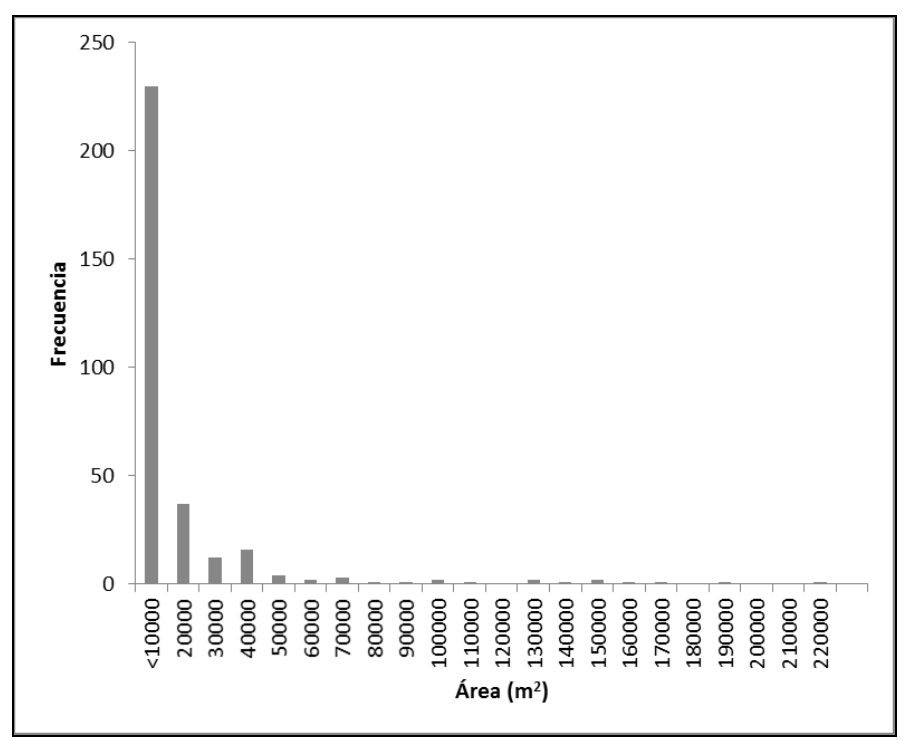

Figura 5- Distribución natural de áreas de las formas estudiadas.

Cuando se calcula la relación entre la superficie total estudiada y la suma de las áreas individuales de las dolinas, se obtiene el Pitting index, que se utiliza comúnmente para estimar el grado de desarrollo del karst, o más bien, la intensidad del proceso de karstificación (White \& White, 1979; Denizman, 2003). Cuando se analiza en términos proporcionales, esto representa un 5,95\% del área de estudio, lo que confirma las consideraciones sobre el gran número de dolinas con pequeñas dimensiones areales. Los valores encotrados por Maldonado et al. (2000) son muy superiores $(21,66 \%)$ a estos aquí presentados, hecho que confirma el nivel de interferencia de la escala de análisis sobre los resultados obtenidos.

Para el estudio de la correlación de los parámetros de Dar-Zarrouk con los atributos morfométricos de las dolinas en una escala de detalle haria falta una gran cantidad de sondeos eléctricos verticales, que el área todavía no dispone.

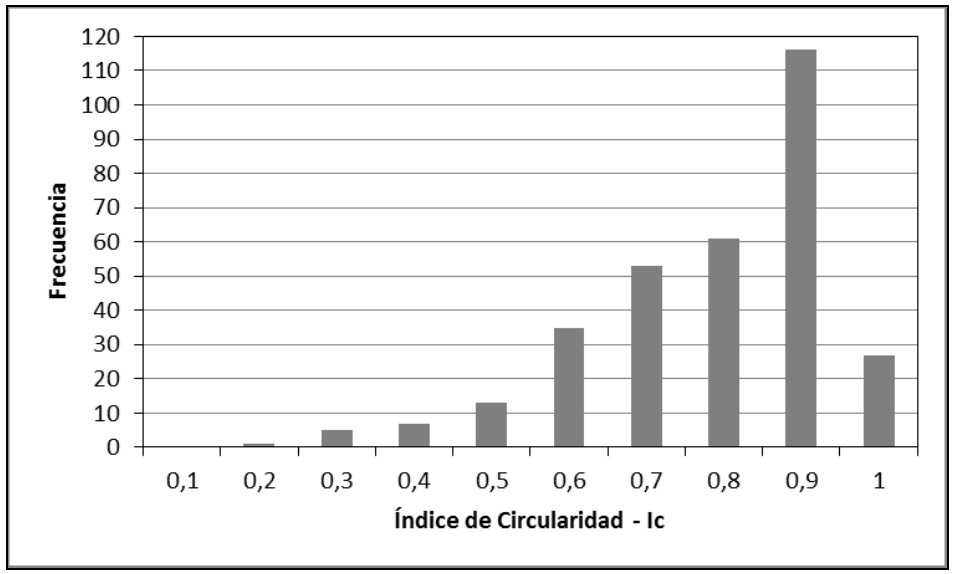

Figura 6- Distribución de los índices de circularidad de las dolinas cartografiadas.

El índice de circularidad de dolinas tiene el objetivo de identificar la similitud entre la geometria de una dolina y la forma perfecta de un círculo. Este índice fue llamado por Mills \& Starnes (1983) índice de irregularidad y puede expresar posibles controles de la estructura geológica sobre la forma de las dolinas. Así como en los estudios morfométricos de cuencas hidrográficas, el cálculo de este índice se da a través de la relación entre el área real de la dolina y el área de un círculo del mismo perímetro que la forma kárstica en cuestión (Williams, 1972). Las dolinas en el área de estudio tienen una distribución asimétrica de este atributo morfométrico (Figura 6), con valores que van desde 0,14 a 0,95 . Esta distribución se explica por una mayor frecuencia de pequeñas dolinas, que tienden a presentarse menos irregular que las grandes formas (Brinkmann et al., 2008). La media y la 
desviación estándar encontrados fueron 0,73 y 0,15 , respectivamente

Otra variable analizada para las dolinas del área en cuestión fue la relación entre la longitud y la anchura, lo que ayuda en la comprensión de los procesos de alargamiento de las formas, destacando el papel desarrollado por las discontinuidades estructurales en el proceso evolutivo de dichas formas kársticas (Maldonado et al., 2000). Los valores encontrados (Figura 7) variaron desde 1,0 hasta 10,39 y la media y desviación estándar fueron de 2,00 y 1,08, respectivamente.

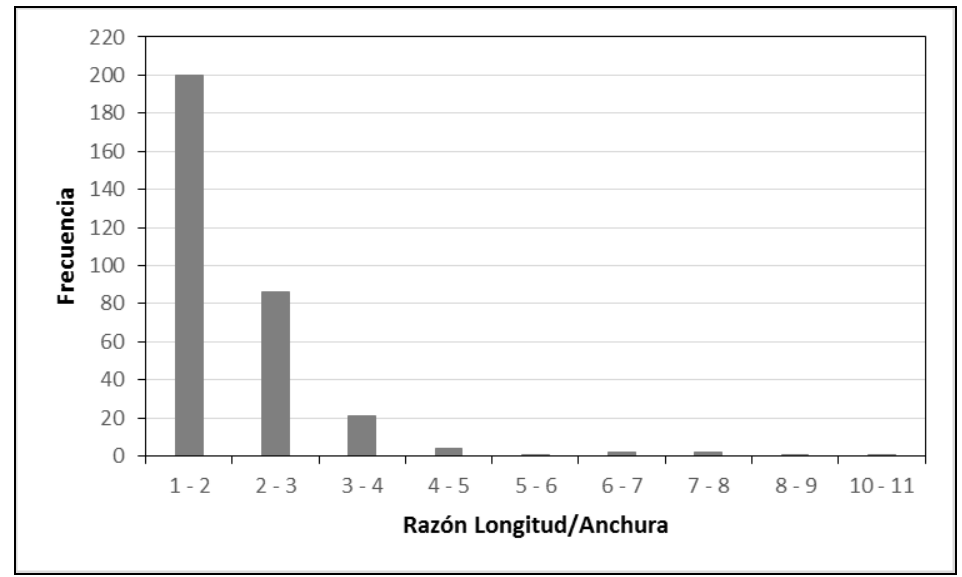

Figura 7 - Distribución natural de la razón longitud/anchura.

Los ejes principales de las 318 dolinas cartografiadas no han puesto de manifiesto el predominio de una dirección NW-SE, aunque se pueda observar una pequeña concentración en la direccione NE-SW (Figura 9). Arlegui y Soriano (1996) reconocieron dos sistemas de diaclasis en esta área, un a la dirección N-S y otro con variaciones NW-SE a WNW-ESE.
Estos valores coinciden con aquellos encontrados por Maldonado et al. (2000) y Gutiérrez et al. (2007), lo que indica que, aunque la densidad de dolinas varía en función de la escala de análisis, la orientación de estas formas no parece ser afectada. Los datos de morfometría levantados en este estudio estan presentados en la tabla 2.

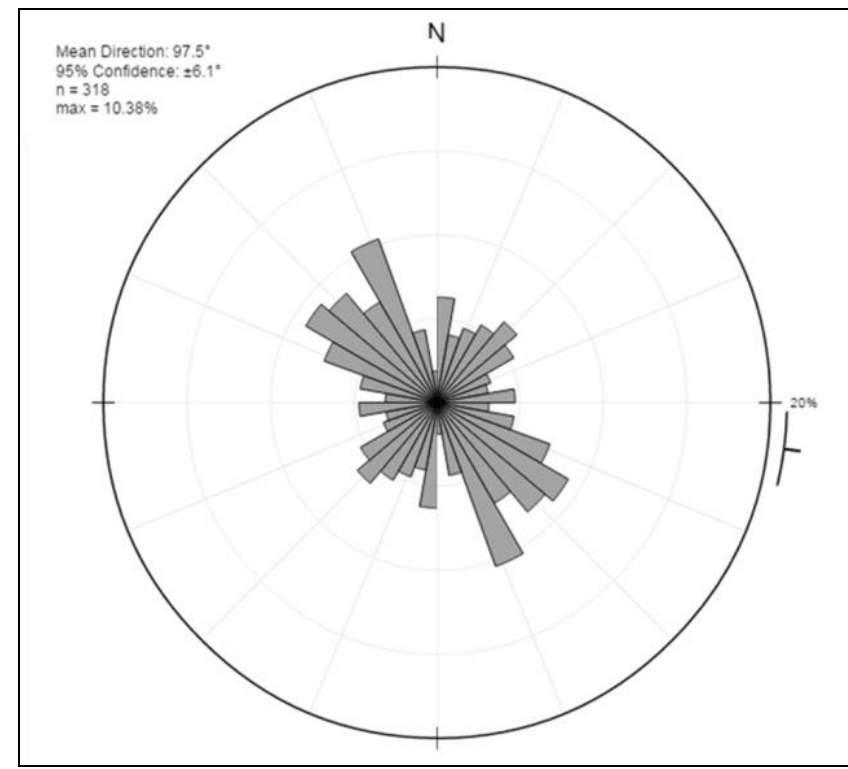

Figura 8 - Orientación de los ejes mayores de las dolinas. El círculo externo corresponde un $20 \%$.

\section{Parámetros de Dar Zarrouk}

Los parámetros de Dar-Zarrouk fueron calculados a partir de los datos de campo de 37 sondeos eléctricos verticales situados en el aluvial del Ebro, aguas abajo de la ciudad de Zaragoza. El número de capas geoeléctricas varió entre 4 y 7 , con media de 5,16 y desviación estándar de 1,0. Los valores de resistividad variaron entre 0,08 y

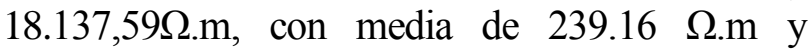

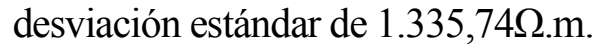


Tabla 2 - Resumen de los datos morfométricos de las dolinas estudiadas.

\begin{tabular}{c|c|c|c|}
\hline Variable & Media & Desviación estándar & Unidad \\
\hline Longitud & 145,87 & 149,85 & $\mathrm{~m}$ \\
Anchura & 73,07 & 78,46 & $\mathrm{~m}$ \\
Area & 13821,95 & $28.850,97$ & $\mathrm{~m}^{2}$ \\
Perímetro & 398,86 & 390,07 & $\mathrm{~m}$ \\
Orientación & 95,95 & 54,32 & Grados \\
Indice de Circularidad & 0,73 & 0,15 & --- \\
Relación Longitud / Anchura & 2,0 & 1,08 & --- \\
\hline
\end{tabular}

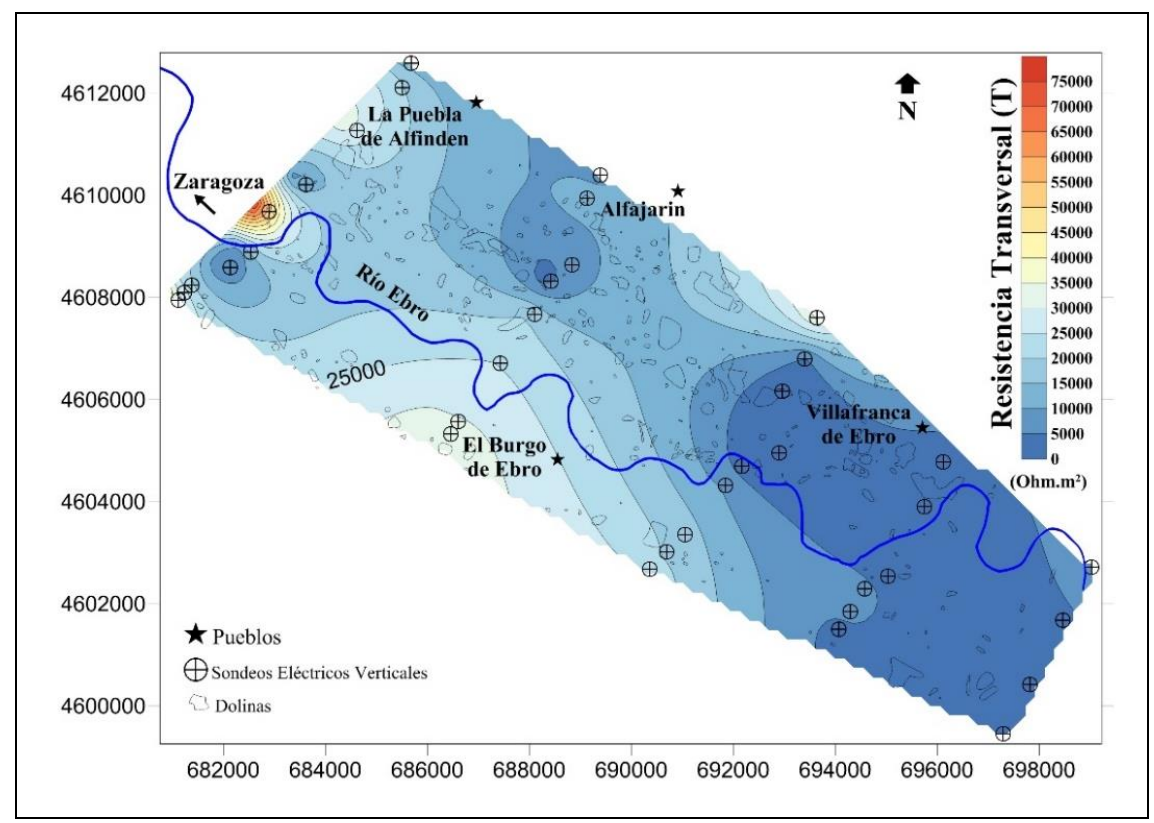

Figura 9 - Mapa de distribución de la resistencia transversal en el área de estudio.

Las curvas predominantes han sido las del tipo HK y HKQ, con 9 y 8 ocurrencias, respectivamente. Los otros tipos de curva fueron observados apenas uno o dos veces. Estos datos exponen el predominio de mayor resistividad en los primeros metros, con una disminución en los valores en función de la profundidad, quizá por la proximidad con el nivel freático.

Los valores de resistencia transversal variaron desde 439,34-78.038,88 $\Omega \mathrm{m}^{2}$, con media $\mathrm{y}$ desviación estándar de $15.320,09 \Omega . \mathrm{m}^{2} \quad \mathrm{y}$ $16.313,88 \Omega . \mathrm{m}^{2}$, respectivamente. La figura 9 muestra la distribución espacial de estos valores, donde se puede observar que las zonas con valores intermedios tienen mayores concentraciones de dolinas, mientras que las zonas con altos niveles de resistencia transversal tiene menor presencia de dichas formas kársticas.

Al analizar la conductancia longitudinal se observa que este parámetro mostró valores que varian entre 0,003 y 0,54 Siemens, con media de 0,08 y desviación estándar de 0,11 Siemens, revelando que las mayores concentraciones de dolinas se encuentran en zonas de media y alta conductancia longitudinal (Figura 10), observadas en la parte media al norte del area.

A pesar de que otros factores pueden afectar esta distribución, una posible explicación para la relación entre la concentración de dolinas y los parámetros Dar-Zarrouk podría ser que en áreas con menor resistencia transversal y mayor conductancia longitudinal, respectivamente, el flujo tiende a ser más efectivo, lo que podría causar mayor disolución de la roca y sedimentos evaporíticos, contribuyendo al desarrollo de las formas kársticas en estas áreas. De hecho, "la conductividad de la roca es proporcional a la conductividad de las aguas subterráneas, lo que es bastante variable, ya que depende de la concentración y el tipo de minerales y sales que contiene disueltos" (Lowrie, 2007, 
p.256). Esta hipótesis puede ser confirmada por los valores de conductividad eléctrica de las aguas subterráneas, que en las áreas con más dolinas apresentan mayor cantidad de sales disueltos (Lamelas, 2007).

Además de la cantidad de formas en estas áreas con valores de resistencia transversal y conductancia longitudinal bajos y elevados, respectivamente, se puede notar la presencia de formas con las dimensiones más grandes del area investigada. Aunque no deba ser adoptada como correspondiente directa de la transmisividad (Frohlich \& Kelly, 1985), los valores de conductancia longitudinal ayudan la definición comparativa de zonas con mayor o menor tasa de migración del flujo subterráneo.

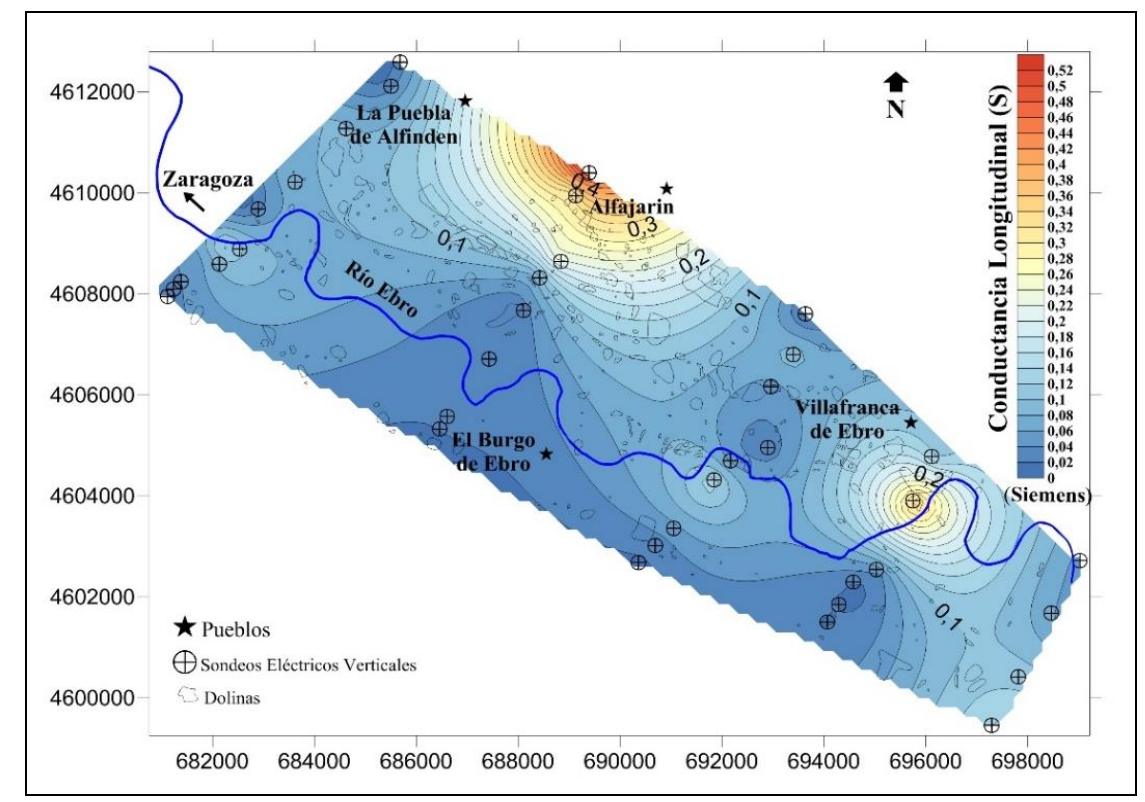

Figura 10 - Mapa de distribución de la conductancia longitudinal en el área de estudio.

\section{CONCLUSIONES}

A partir de los levantamientos llevados a cabo en una zona con gran cantidad de dolinas aguas abajo de la ciudad de Zaragoza, fue posible establecer una relación entre los datos de fracturas e fallas geológicas regionales y las características morfométricas de las formas kársticas. El predomínio de dolinas pequenãs, a menudo, constituye un problema para la planificación de ocupación y la prevención de los desastres naturales, dado que a menudo las pequeñas formas suelen ser olvidadas en términos de riesgos geológicos.

La adopción de los parámetros de Dar-Zarrouk resultó satisfactoria para la investigación de las posibles relaciones entre las direcciones del flujo hídrico y la concentración de dolinas en zonas de sedimentos evaporíticos.

También hay una falta de estudios estructurales en escala de detalle, apuntando a la correlación entre los factores geológicos, tales como fallas y fracturas, y las zonas de concentración de dolinas. Sin embargo, algunos trabajos en curso ya están utilizando imágenes LIDAR para una mejor comprensión de las formas kársticas en el área de estúdio, poniendo de relieve la importancia de la teledetección para estudios de este tipo.

Los intentos de correlación entre parámetros geofísicos y desarrollo de formas kársticas se vuelve muy importante en zonas con limitaciones naturales al proceso de urbanización acelerada corriente que las medianas y grandes ciudades han sufrido este siglo. La comprensión de estas características naturales del terreno puede prevenir la degradación de los recursos hídricos subterráneos y también apuntar áreas más propícias al desarrollo de colapsos kársticos, eminentemente peligrosos a la dinámica urbana.

\section{AGRADECIMIENTOS}

Este trabajo ha sido realizado gracias a colaboración de la institución brasileña CAPES que, por medio del Programa Ciências Sem Fronteiras, ha concedido beca de post-doctorado al Dr. Fernando de Morais (Proceso n.1079-01-3). Además, los autores quieren agradecer la Confederación Hidrográfica del Ebro por tener disponibilizado los datos de sondeos eléctricos verticales. 


\section{BIBLIOGRAFÍA}

AHMED, S. \& CARPENTER, P.J. Geophysical response of filled sinkholes, soil pipes and associated bedrock fractures in thinly mantled karst, east-central Illinois. Environmental Geology, v. 44, n. 6, p. 705-716, 2003.

ARANDJELOVIC, D.A possible way of tracing groundwater flows in karst. Geophysical Prospecting, v. 17, n. 4, p. 404418, 1969.

ARANDJELOVIC, D. Geophysical methods used in solving some geological problems encountered in construction of the Treblinisca water power plant (Yugoslavia). Geophysical Prospecting, v. 14, n. 1, p. 80-97, 1966.

ARLEGUI, L.E. \& SORIANO, A. Lineamientos y su influencia en los modelados del centro de la cuenca del Ebro. In: A. GRANDAL D'ANGLADE \& J. PAGÉS VALCARLOS (Coordinadores), IV Reunión Nacional de Geomorfología, Cadernos do Laboratorio Xeolóxico de Laxe, v. 21, p. 11-21, 1996.

BECK, B. A generalized genetic framework for the development of sinkholes and karst in Florida, U.S.A. Environmental Geology and Water Sciences, v. 8, n. 1-2, p. 5-18, 1986.

BENITO, G. Karstificación y colapsos kársticos en los yesos del sector central de la Depresión del Ebro. Cuaternario y Geomorfología, v.1, p. 61-76, 1987.

BENITO, G. \& GUTIÉRREZ, M. Karst in Gypsum and its Environmental Impact on the Middle Ebro Basin, Spain. Environmental Geology and Water Sciences, v. 12, n. 2, p. 107-111, 1988

BENITO, G.; PEREZ DE CAMPO, P.; GUTIÉRREZELORZA, M.; SANCHO, C. Natural and Human induced sinkholes in gypsum terrain and associated environmental problems in NE Spain. Environmental Geology, v. 25, n. 3, p. 156-164, 1995.

BONDESAN, A.; MENEGHEL, M.; SAURO, U. Morphometric analysis of dolines. International Journal of Speleology, v. 21, n. 1-4, p. 1-55, 1992.

BRINKMANN, R.; PARISE, M.; DEY, D. Sinkhole distribution in a rapidly developing urban environment: Hillsborough County, Tampa Bay area, Florida. Engineering Geology. v. 99, n. 3-4, p. 169-184, 2008.

CARBONEL, D.; RODRÍGUEZ, V.; GUTIÉRREZ, F.; MCCALPIN, J.P.; LINARES, R.; ROQUÉ, C., ZARROCA, M.; GUERRERO, J.; SASOWSKY, I. Evaluation of trenching, ground penetrating radar (GPR) and electrical resistivity tomography (ERT) for sinkhole characterization. Earth Surface Processes and Landforms, v. 39, n. 2, p. 214-227, 2014.

CVIJIĆ, J. DAS Karstphänomen. Versuch einer morphologischen Monographie- Geographische Abhandlungen, Bd. V. Heft. Wien, v.3, p. 1-114, 1893.

DAY, M. The morphology and hydrology of some Jamaican karst depressions. Earth Surface Processes and Landforms, v. 1, p. 111-129, 1976.

DE WAELE,J.; PLAN,L.; AUDRA,P. Recent developents in surface karst geomorphology. Geomorphology, v. 106, n. 12, p. 1-8, 2009.

DENIZMAN, C. Morphometric and spatial distribution parameters of karstic depressions, lower Suwannee River Basin, Florida. Journal Cave Karst Studies, v. 65, n. 1, p. 29-35, 2003.

FORD, D.C. \& WILLIAMS, P.W. Karst Geomorphology and Hydrology. Unwin Hyman, London, 601 p., 1989.

FROHLICH, R.K \& KELLY, W.E. The relation between hydraulic transmissivity and transverse resistance in a complicated aquifer of glacial outwash deposits. Journal of Hydrology, v. 79, n. 3-4, p. 215-229, 1985.

GALVE,J.P.; GUTIÉRREZ, F.; LUCHA, P.; BONACHEA, J.;
CENDRERO, A.; GIMENO, M.J.; GUTIÉRREZ, M.; PARDO, G.; REMONDO, J.; SÁNCHEZ, J.A. Sinkholes in the salt-bearing evaporate karst of the Ebro River valley upstream of Zaragoza city (NE Spain). Geomorphological mapping and analysis as a basis for risk management. Geomorphology, v. 108, n. 3-4, p. 145-158, 2009.

GIL, H.; PEPE, M.; SORIANO, M.A.; PARISE, M.; POCOVÍ, A.; LUZÓN, A.; PÉREZ, A.; BASSO, A. Sviluppo ed evoluzione di sprofondamenti in rocce solubili: um confronto tra il carso coperto del Bacino dell'Ebro (Spagna) e la Penisola Salentina (Italia).- Memorie Descrittive Carta Geologica d'Italia, v. 93, p. 253-276, 2013.

GIL, H.; LUZÓN, A.; SORIANO, M.A.; PUEYO, O.; POCOVÍ, A.; PÉREZ, A. Ejemplos de formas paleokársticas y depósitos asociados en los materiales detríticos de terraza del río Ebro. Geogaceta, v. 52, p. 97-100, 2012.

GUTIÉRREZ, F.; CALAFORRA, J.M.; CARDONA, F.; ORTÍ, F.; DURÁN, J.J.; GARAY, P. El karst em las formaciones evaporíticas españolas. In. Andreo, B., DURÁN, J.J. (Eds.). Investigaciones en sistemas kársticos españoles. IGME, Madrid, p. 49-87, 2004.

GUTIÉRREZ, F.; GALVE, J.P.; GUERRERO, J.; LUCHA, P.; CENDRERO, A.; REMONDO, J.; BONACHEA, J.; GUTIERREZ, M.; SANCHEZ, J.A. The origin, typology, spatial distribution and detrimental effects of the sinkholes developed in the alluvial evaporite karst of the Ebro River valley downstream of Zaragoza City (NE Spain). Earth Surface Processes and Landforms, v. 32, n.6, p. 912-928, 2007.

GUTIÉRREZ, F.; GUERRERO, J.; LUCHA, P. A genetic classification of sinkholes illustrated from evaporite paleokarst exposures in Spain; Karst processes, landforms, and environmental problems. Environmental Geology, v. 53, n. 5, p. 993-1006, 2008.

GUTIÉRREZ-SANTOLALLA， F.; GUTIÉRREZ-ELORZA, M.; MARÍN, C.; MALDONADO, C.; DESIR, G. Spatial distribution, morphometry and activity of La Puebla de Alfindén sinkhole field in the Ebro river valley (NE Spain): applied aspects for hazard zonation. Environmental Geology, v. 48, n. 3, p. 360-369, 2005.

JENNINGS, J.N. Karst geomorphology. 2 Ed., Oxford: Basil Blackwell, 293 p., 1987.

KELLY, W.E. Geoelectric sounding for delineating ground-water contamination. Ground Water, v. 14, n. 1, p. 6-10, 1976.

KELLY, W.E. Geoelectric sounding for estimating aquifer hydraulic conductivity. Ground Water, v. 15, n. 6, p. 420-425, 1977.

KOSINSKI, W.K. \& KELLY, W.E. Geoelectric soundings for predicting aquifer properties. Ground Water, v. 19, n. 2, p. 163-171, 1981.

KRUSE, S.; GRASMUECK, M.; WEISS, M.; VIGGIANO, D. Sinkhole structure imaging in covered karst terrain. Geophysical Research Letters, v. 33, n. L16405, 2006.

LAMELAS, M.T. Geo-resources and geo-hazards in the context of a sustainable development in the periphery of urban areas, exemplary of a part of the Ebro Basin in the surroundings of Zaragoza (Spain). Zaragoza, 2007. 211 p. $\mathrm{PhD}$ thesis, Universidad de Zaragoza.

LAND, L. Geophysical records of anthropogenic sinkhole formation in the Delaware Basin region, Southeast New Mexico and West Texas, USA. Carbonates and Evaporites, v. 28, n. 1-2, p. 183-190, 2013.

LOWRIE, W. Fundamentals of Geophysics. Second edition. Cambridge University Press, Cambridge, UK, 381 p., 2007.

LUZÓN, A.; RODRÍGUEZ-LÓPEZ, J.P.; PÉREZ, A.; SORIANO, M.A.; GIL, H.; POCOVÍ, A. Karst subsidence as a control on the accumulation and preservation of aeolian deposits: A Pleistocene example from a proglacial outwash 
setting, Ebro Basin, Spain. Sedimentology, v. 59, n. 7, p. 2199-2225, 2012.

LUZÓN, A.; PÉREZ, A.; SORIANO, M. A.; POCOVÍ, A. Sedimentary record of Pleistocene paleodoline evolution in the Ebro basin (NE Spain). Sedimentary Geology, v. 205, n. 1-2, p. 1-13, 2008.

MAILLET, R. The fundamental equations of electrical prospecting. Geophysics, v. 12, n. 4, p. 529-556, 1947.

MALDONADO, C.; GUTIÉRREZ-SANTOLALLA, F.; GUTIÉRREZ-ELORZA， M.; DESIR， G. Distribución espacial, morfometría y actividad de la subsidencia por disolución de evaporitas en un campo de dolinas de colapso (Valle del Ebro, Zaragoza). Cuaternario y Geomorfología, v. 14, n. 3-4, p. 9-24, 2000.

MILANOVIC, P.T. Water Resources Engineering in Karst. CRC Press, Boca Raton, 312 p., 2004.

MILLS, H.H. \& STARNES, D.D. Sinkhole morphometry in a fluviokarst region: eastern Highland Rim, Tennessee, U.S.A. Zeitschrift für Geomorphologie, v. 27, n. 1, p. 93-54, 1983.

MUÑOZ, A.; ARENAS, C.; GONZÁLEZ, A.; LUZÓN, A.; PARDO, G.; PÉREZ, A.; VILLENA, J. Ebro Basin (northeastern Spain). In: GIBBONS, W. \& MORENO, T. (Coordinadores). The Geology of Spain. Geological Society of London, London, p. 370-385, 2002.

NIWAS, S. \& SINGHAL, D.C. Estimation of aquifer transmissivity from Dar-Zarrouk parameters in porous media. Journal of Hydrology, v. 50, p. 393-399, 1981.

NIWAS, S. \& SINGHAL, D.C. Aquifer transmissivity of porous media from resistivity data. Journal of Hydrology, v. 82, n. 1-2, p. 143-153, 1985.

NIWAS, S.; TEZKAN, B.; ISRAIL, M. Aquifer hydraulic conductivity estimation from surface geoelectrical measurements for Krauthausen test site, Germany. Hydrogeology Journal, v. 19, n. 2, p. 307-315, 2011.

OCTAVIO DE TOLEDO, F. Estudio del aluvial del Ebro en la Província de Zaragoza. Ministerio de Medio Ambiente y Medio Rural y Marino, Gobierno de España. 24 p., 1989.

OGDEN, A.E. Morphometric Analysis of the Sinkholes in the Greenbrier Limestone of West Virginia, In: PROCEEDINGS OF 2ND ENVIRONMENTAL PROBLEMS IN KARST TERRANES AND THEIR SOLUTIONS, p. 29-49, 1988.

ORELlANA, E. Prospeccion geoelectrica en corriente continua. Ed. Paraninfo, 523 p., 1972.

PALMQUIST, R. Geologic controls on doline characteristics in mantled karst. Zeitschrift für Geomorphologie Suppl., v. 32, p. 90-106, 1979.

PARDO, G.; ARENAS, C.; GONZÁLEZ, A.; LUZÓN, A.; MUÑOZ, A.; PÉREZ, A.; PÉREZ-RIVARÉS, F.J.; VÁZQUEZ-ÚRBEZ, M.; VILLENA, J. La Cuenca del Ebro. Geología de España. IGME, p. 533-543, 2004.

PUEYO-ANCHUELA, O.; CASAS-SAINZ, A.M.; SORIANO, M.A.; POCOVÍ-JUAN, A. A geophysical survey routine for the detection of doline areas in the surroundings of Zaragoza NE Spain. Engineering Geology, v.114, p. 382-396, 2010.

REYNOLDS, J.M. An Introduction to Applied and Environmental Geophysics, Second edition. WileyBlackwell, John Wiley and Sons, Itd, Chichester, UK, 710 p., 2011.

RIBA, O.; REGUANT, S.; VILLENA, J. Ensayo de sintesis estratigráfica y evolutiva de la Cuenca terciaria del Ebro. Libro jubilar J. M. Rios. Geología de España II. IGME, Madrid, 131-159, 1983.

SAURO, U. Dolines and sinkholes: aspects of evolution and problems of classification. Acta Carsologica, v. 32, n. 2, p. 41-52, 2003.
SINCLAIR, W.C., STEWART, J.W., KNUTILLA, R.L., GILBOY, A.E., MILLER, R.L. Types, Features, and Occurrence of Sinkholes in the Karst of West-Central Florida. U.S. Geological Survey Water Resources Investigations Report, 85-4126, 1985.

SINGH, U.K.; DAS, R.K.; HODLUR, G.K. Significance of Dar-Zarrouk parameters in the exploration of quality affected coastal aquifer systems. Environmental Geology, v. 45, n. 5, p.696-702, 2004.

SORIANO, M.A. \& SIMÓN, J.L. Alluvial dolines in the central Ebro basin, Spain: a spatial and developmental hazard analysis. Geomorphology, v. 11, n. 4, p. 295-309, 1995.

SORIANO, M.A. Geomorfología del sector centromeridional de la Depresión del Ebro. Publicación 1231, Institución Fernando el Católico, Zaragoza, 269 p., 1990.

SORIANO, M.A. Characteristics of the alluvial dolines developed because of gypsum dissolution in the central Ebro Basin. Zeitschrift für Geomorphologie Suppl., v.85, p.59-72, 1992.

SORIANO, M.A.; SIMÓN, J.L.; GRACIA, J.; SALVADOR, M. Alluvial sinkholes over gypsum in the Ebro basin (Spain): genesis and environmental impact. Hydrological Sciences Journal, v. 39, n. 3, p. 257-268, 1994.

SRINIVAS, Y.; OLIVER, H.D.; RAJ, A.S.; MUTHURAJ, D.; CHANDRASEKAR, N. Estimation of conductance anomalies in subsurface through Dar-Zarrouk parameters by resistivity inversion method. International Journal of Physical and Mathematical Sciences, v. 3, n. 1, p. 140-151, 2012.

STEVANOVIC, Z. \& DRAGISIC, V. An example of identifying karst groundwater flow. Environmental Geology, v. 35, n. 4, p. 241-244, 1998.

STIERMAN, D.J. Geophysical detection of caves and karstic voids. In: Encyclopedia of Caves and Karst Science (ed. J. Gunn), Fitzroy Dearborn, New York, p. 377-380, 2004.

SUMMERFIELD, M.A. Global Geomorphology: an introduction to the study of landforms. Harlow - England: Pearson Prentice Hall. 537 p., 1991.

SWEETING, M. M. Karst Landforms, The Macmillian Press, London. 1972.

VALOIS, R.; CAMERLYNCK, C.; DHEMAID, A.; GUERIN, R.; HOVHANNISSIAN, G.; PLAGNES, V.; REJIBA, F.; ROBAIN, H. Assessment of doline geometry using geophysics on the Quercy plateau karst (South France). Earth Surface Processes and Landforms, v. 36, p.1183-1192, 2011.

VERESS, M. Investigation of covered karst form development using geophysical measurements. Zeitschrift für Geomorphologie, v. 53, n. 7, p. 467-486, 2009.

WHITE, E.L. \& WHITE, W.B. Quantitative morphology of landforms in carbonate rock basins in the Appalachian Highlands. Geological Society of America Bulletin, v. 90, p. 385-396, 1979.

WILLIAMS, P.W. Illustrating morphometric analysis of karst with examples from New Guinea. Zeitschrift für Geomorphologie, v. 15, p. 40-61, 1971.

WILLIAMS, P.W. Morphometric analysis of polygonal karst in New Guinea. Geological Society of America Bulletin, v. 83, p. 761-796, 1972.

WILLIAMS, P.W. The role the subcutaneous zone in karst hydrology. Journal of Hydrology, v. 61, n. (1/3), p.45-67, 1983.

Manuscrito recebido em 22 de outubro de 2015 Revisado e aceito em 31 de outubro de 2016 